\title{
Steady-state analysis of a natural gas distribution network with hydrogen injection to absorb excess renewable electricity
}

\author{
Lapo Cheli", Gabriele Guzzo, Dominique Adolfo, Carlo Carcasci
}

DIEF: Department of Industrial Engineering, University of Florence, Via Santa Marta, 3, 50139, Florence, Italy

\section{H I G H L I G H T S}

- Gas distribution networks can absorb the renewable energy surplus through hydrogen injection.

- Steady-state simulations of a gas distribution network during a winter day in Tuscany.

- Critical nodes analysis in terms of fluid-dynamics parameters.

- Critical nodes pointed out by Chromatic Maps of the Wobbe Index.

- Necessity of injection control logic at peak of renewable energy production.

\section{A R T I C L E I N F O}

Article history:

Received 22 February 2021

Received in revised form

13 May 2021

Accepted 13 May 2021

Available online 8 June 2021

Keywords:

Hydrogen

Gas distribution networks

Power-to-Gas

Natural gas

Sector coupling

Wobbe index

\section{G R A P H I C A L A B S T R A C T}

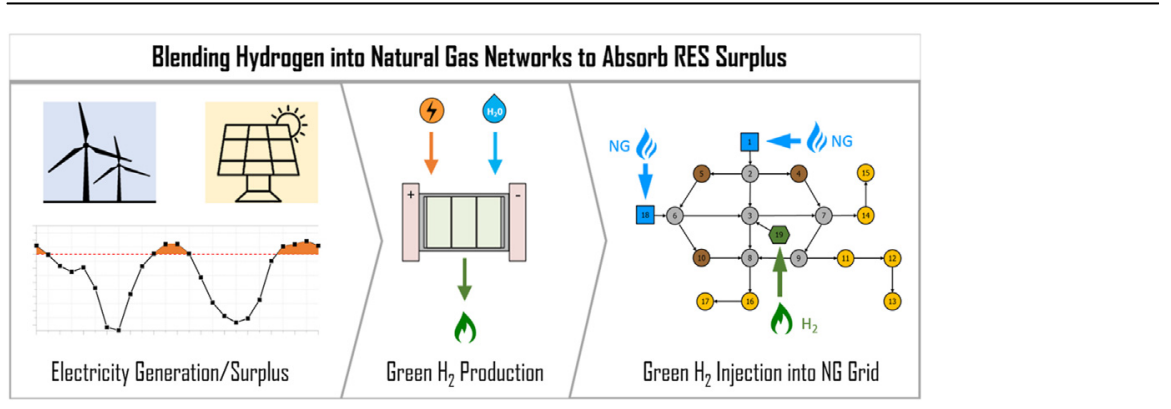

\footnotetext{
* Corresponding author.

E-mail addresses: lapo.cheli@unifi.it (L. Cheli), gabriele.guzzo@unifi.it (G. Guzzo), dominique.adolfo@unifi.it (D. Adolfo), carlo.carcasci@unifi.it (C. Carcasci). 
power production, leads to an amplification of the $\mathrm{H} 2$ injection impact and hence to a reduction of the Wobbe Index value that overcomes the safety lower limit.

(c) 2021 Hydrogen Energy Publications LLC. Published by Elsevier Ltd. All rights reserved.

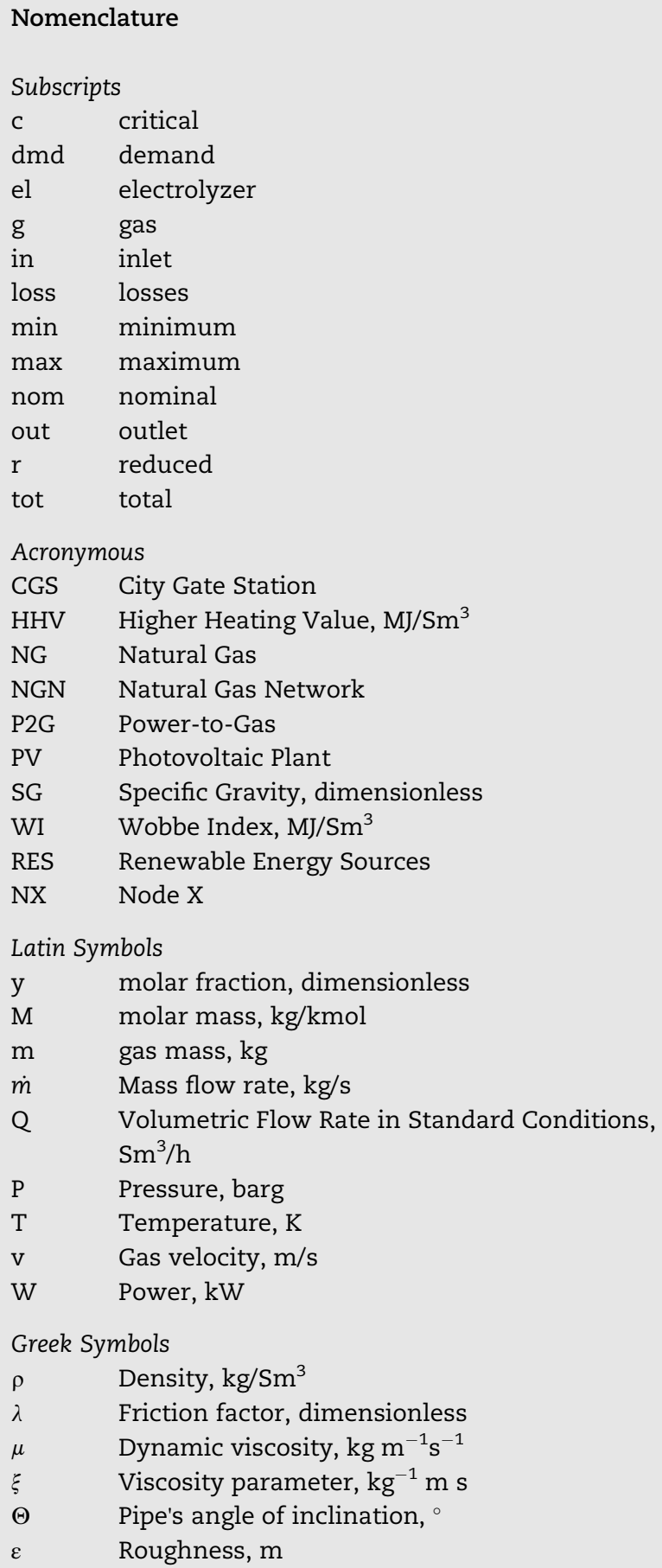

\section{Introduction}

Natural gas has been identified as the fuel for the energy transition of the 21st century according to several institutions and scientists; in fact, it has the lowest emission factor if compared with the other two main fossil fuels used for power and thermal production (i.e. coal and oil) [1]. According to many studies, the natural gas distribution network will remain present and active for the next thirty years but it will have to evolve over that period [2]. The IEA predicts that NG will meet about $25 \%$ of primary energy demand by 2050 but the gas grid will have to manage a series of alternative gases produced from renewable sources, in particular biomethane and green hydrogen; focusing on the latter one, to support the transition of the grid, some pipelines will be reconditioned to be able to transport a higher percentage of hydrogen until dedicated lines will be created to transport $100 \%$ of hydrogen [2-4].

\section{P2G projects}

Power-to-gas (P2G) systems need a surplus of electricity to feed an electrolyser which generates a chemical energy carrier in gaseous form (i.e., hydrogen); the produced gas is called "green" if the electrolyser absorbs the excess of production from renewable energy sources, such as PV solar energy or wind energy. Hydrogen can be used to decarbonize lots of industrial or energy processes or as an energy vector for mobility [5,6]. Gahleitner summarized the most important power-to-gas pilot plants in Europe [7], that have either already been realized or are being planned. Qadrdan et al. presented an in-depth analysis of the role of the power-to-gas application in an integrated gas and electricity system at regional and national level [8].

Moreover, a huge power to gas project that have to be mentioned is the NATURALHY EU Project with more than 60 partners coordinated to study the effect of injecting hydrogen into the natural gas network: [9].

An additional strand of the use of hydrogen produced by P2G systems is to inject it directly, or after its conversion in Synthetic Natural Gas (SNG) [10], into the existing natural gas network with several advantages: immediate reduction of the overall greenhouse gas emissions from the gas grid and consequential decarbonization, energy systems flexibility increasing through the gas grid which acts as renewable electricity buffer when RES produce an excess of energy and which releases the energy when an excess of demand occurs $[11,12]$. The main constrain of the injection in the natural gas 
grid is that the hydrogen feed-in has to be limited to certain percentages in order to maintain the properties of the resulting gas mixture (i.e. heating value, Wobbe Index, etc ...) within the national standard values $[9,13]$.

Studies of the risks and on the feasibility of using a designed natural gas network with a blended hydrogen mixture or with hydrogen gas only have been already conducted deeply, here are some papers for the reader's additional investigation [6,14-21].

Simonis et. all presented a study of the sizing of a P2G system used to absorb excess wind energy production and its injection in the nearest urban gas distribution network in the form of green hydrogen and synthetic methane allowing the transition from medium to high renewable power penetration for urban regions [22].

With the soaring diffusion of renewable energies and the development in parallel of P2G technologies, the interest within the scientific world to model and simulate the injection of hydrogen into the natural gas network has arisen. The goal is to investigate and quantify the beneficial effects and the main technical constraints. In literature, several recent works studied gas pipelines and gas distribution networks' behaviour with natural gas only [23], but also in the presence of hydrogen or alternative gas injections [1,17,24-27].

\section{H2 - NG blending literature review}

This section presents the main articles dealing with the analysis and simulation of hydrogen injection into the natural gas network.

Pellegrino et al. developed an NGN model under steadystate condition, adopting a non-isothermal approach. Complex pipeline networks have been modelled (with gas compression stations and other non-pipe elements) and the results have shown that the proposed tool can simulate the distributed injection of green fuel gases (e.g., hydrogen and substitute natural gas, etc.) into the NGN taking into account a various group of gas mixture compounds (methane, ethane, hydrogen and so on ...) and also to capture the effect of multiple gas source injection (e.g., shale gas, LNG, biomass-based supplies) on existing gas networks [1].

Mazza et. all developed a representative urban natural gas network to evaluate the feasibility of coupling the natural gas network to the power grid through a power-to-gas systems producing green hydrogen and injecting it into the gas grid, assessing its performances in terms of gas quality indexes [24].

Guandalini et al. developed an unsteady model of a gas pipeline portion to assess the dynamic effects of hydrogen injection and blending in the gas network. A comparison was made between a case of NG-only and a case of hydrogen mixed with NG for the same network. The analysis showed effects on the higher heating value, Wobbe index, and density that are not negligible, but within a quality assurance range; furthermore, it was shown that the allowed hydrogen fractions are limited and very sensitive to the applied boundary conditions such as the profile of the users and the size of the connected gas supply stations [25].

Chaczykowski et all. Investigates two methods for tracking alternative gases (i.e., biomethane and hydrogen) and natural gas mixtures composition, one based on using a moving grid method, and one solving the advection equation. These methods were applied to one Polish gas pipeline and one Norwegian offshore gas transmission system and the results showed well-measured compositions and transport times, with an error in total transport times of less than 2.0\% [26].

Abeysekera et. all studied a theoretical urban network with low-pressure hydrogen and biomethane (upgraded biogas) injections in the gas grid: a steady-state model was presented using two different approaches: the first imposes the gas volumetric flow rate requested by users, while the second method tracks the users' energy request and increases the flow where is necessary: results show that the injection of alternative gases, such as hydrogen, in the gas network can affects the quality index and the fluid-dynamics of the grid, in particular the gas flow velocity and pressure, but if accurately managed, the hydrogen blending can help to control the power grid and reduce the natural gas importations $[27,28]$.

Colbertaldo et. all and Pellegrino et. all analysed the feasibility of injecting hydrogen into the Italian natural gas grid: the former highlighted the role of hydrogen and powerto-gas systems considering the national energy mix by 2050 and the expected percentage of renewables penetration; the latter conducted a preliminary study to evaluate the effects of a $10 \%$ injection of hydrogen into the overall natural gas mix transported by the Italian grid and consumed in the country. The article pointed out that the Italian gas network could already accommodate about 715,000 Sm3/year green hydrogen blending, corresponding to an installed capacity of about 78MWof electrolysers [29,30].

\section{Motivation}

Considering the studies presented so far, it is still necessary to carry out detailed steady-state analyses of a gas grid under hydrogen injections. Even if based on simplifying hypotheses, an hourly variation of daily demand already enables to see the effects on a gas network and the main constraints of the hydrogen injection, also considering the different load profiles of the users during the day. For this reason, this article fits into this line of research and seeks to extend the studies carried out so far.

In this article, the steady state-analysis of a local natural gas distribution network is presented: the considered network is used to store the surplus of renewable energy produced by renewable plants operating nearby and the effects of blending are also quantified and discussed. The possibility to inject the RES-produced hydrogen in the natural gas distribution network has the twofold advantage of not transporting the renewable energy produced on-site over long distances and allows to simplify the P2G system, which is typically accompanied also by a compressor-heat-exchanger unit for feeding the gas into the high-pressure grid [31].

The surplus of renewable energy is injected into the gas network in the form of hydrogen, which was previously produced using an electrolyser; a P.E.M. type device is chosen from among the various technologies on the market. In this work a simulation campaign has been carried out throughout a typical average winter day where gas demand is high, choosing Tuscany, in central Italy, as the geographical 
location of the city's network. The wind and solar power production are calculated using wind load and irradiation curves typical of the region $[32,33]$ while the gas and electricity consumption of the users connected to the gas network has been assumed, taking care to match the electrical and gas requirements. The main gas network constraints were analysed under the aforementioned conditions.

\section{Materials and methods}

This chapter is divided into two sections: in the first paragraph the case study is presented, whereas in the second part the authors describe the gas networks steady model; the case study, a simple urban gas grid coupled with a P2G system, has been conceptualized and designed with a few numbers of nodes and pipes, with the first purpose of rapidly highlight and point out the effects of hydrogen injections in terms of thermo-fluid-dynamics impacts and injection position sensitivity. Moreover, the presence of two different classes of users (residential and industrial consumers) and two different RES (PV and wind) has allowed us to understand the gas grid behaviour under distributed hydrogen injections during a typical winter day.

\section{Case study}

The reference gas grid, as already presented by Adolfo D. and Carcasci C. in their previous work [34], is a medium pressure distribution network, composed of two CMS ("Control and Measuring Stations") which deliver the natural gas from the regional medium pressure pipelines into the medium pressure network and supply it to industrial and residential nodes; these nodes represent aggregate gas demand clusters of users and the values of the required maximum daily flow rate are presented in Table 1.

The natural gas is provided at a relative pressure of 0.50 barg and a temperature of $15^{\circ} \mathrm{C}$. The reference gas distribution network layout is presented in Fig. 1.

As specified by the Italian regulations for 6th species gas pipelines [35], the following constraints are applied: operating pressure of the fluid between 0.04 barg and 5 barg and a maximum gas velocity of the fluid of $15 \mathrm{~m} / \mathrm{s}$. The P2G system considered consists of the following technologies: PV Array, Wind Farm, and P.E.M. electrolyser, as you can see in the
Table 2 - RES technologies features for the case study.

\begin{tabular}{lc} 
Technology & $\mathrm{W}_{\mathrm{p}}[\mathrm{MW}]$ \\
\hline PV Array & 5.5 \\
Wind Farm & 3 \\
\hline
\end{tabular}

schematic diagram in Fig. 2. The PV and Wind farm peak sizes are enlisted in Table 2.

In Fig. 3 we can see the daily common dimensionless load demand profiles (gas and electricity) for two different typologies of users: industrial and residential. The demand profiles used in this work are obtained from Refs. [26,35-38].

- Gas demand profiles: a typical residential user demand profile consists of three different peak zones; those are in correspondence with the three main meals (gas for cooking and heating in the evening). A common industrial user is characterized by a constant demand for gas from 8:00. To 18:00 during a working day.

- Electricity demand profiles: a typical profile of a domestic user is characterized by a dynamic evolution over the day. While the baseline is always maintained by the equipment to produce cold (refrigerators and freezers) the demands for electricity increase during the day (decreasing only during lunchtime), reaching the peak around dinner time (19:00-21:00). Then it starts to decrease until the night hours are reached. For an industrial user, the pattern is like the gas one, with a difference regarding the demand peak (expected at 07:00) and with a higher variability due to the presence of electrical equipment.

As a residential user in the considered climate zone, a typical house of $3 \mathrm{kWp}$ and $90 \mathrm{~m}^{2}$ with an annual consumption of $2700 \mathrm{kWh}$ for electricity and annual consumption of about $114.1 \mathrm{kWh} / \mathrm{m}^{2}$ year of Natural Gas has been assumed [36]. Due to the enormous variability of the ratio between thermal and electrical consumption for an industrial user, a reference value has been chosen to maintain the approach as general as possible. Based on the assumption of considering only micro and small factories present in the area, average annual consumption of about $26,000 \mathrm{Sm}^{3}$ for natural gas and $100 \mathrm{MWh}$ for electricity has been assumed [39] in order to match gas and electricity demand and derive a representative daily gas demand curve correspondingly. Considering a

\begin{tabular}{|c|c|c|c|c|c|c|c|c|c|}
\hline Node & $\mathrm{Q}_{\max }\left[\mathrm{Sm}^{3} / \mathrm{h}\right]$ & Pipe & $\mathrm{D}_{\text {pipe }}[\mathrm{m}]$ & $\mathrm{L}_{\text {pipe }}[\mathrm{m}]$ & Species & Pipe & $\mathrm{D}_{\text {pipe }}[\mathrm{m}]$ & $\mathrm{L}_{\text {pipe }}[\mathrm{m}]$ & Species \\
\hline 4 & 230.00 & $1-2$ & 0.16 & 200 & 6 th & $8-9$ & 0.11 & 350 & 6 th \\
\hline 5 & 180.00 & $2-3$ & 0.16 & 500 & 6th & $8-10$ & 0.11 & 350 & 6th \\
\hline 10 & 210.00 & $2-4$ & 0.11 & 350 & 6 th & $9-11$ & 0.08 & 100 & 6 th \\
\hline 11 & 50.00 & $2-5$ & 0.11 & 350 & 6 th & $11-12$ & 0.08 & 100 & 6 th \\
\hline 12 & 75.00 & $6-3$ & 0.16 & 500 & 6th & $12-13$ & 0.08 & 100 & 6th \\
\hline 13 & 35.00 & $3-7$ & 0.16 & 500 & 6 th & $7-14$ & 0.08 & 100 & 6 th \\
\hline 14 & 45.00 & $3-8$ & 0.16 & 500 & 6 th & $14-5$ & 0.08 & 100 & 6 th \\
\hline 15 & 90.00 & $6-5$ & 0.11 & 522 & 6 th & $8-16$ & 0.08 & 100 & 6 th \\
\hline 16 & 65.55 & $7-4$ & 0.11 & 522 & 6 th & $16-17$ & 0.08 & 100 & 6 th \\
\hline \multirow[t]{2}{*}{17} & 55.00 & $6-10$ & 0.11 & 522 & 6 th & $18-6$ & 0.16 & 200 & 6 th \\
\hline & & $7-9$ & 0.11 & 522 & 6 th & 19-Ninj & 0.16 & 283 & 6 th \\
\hline
\end{tabular}




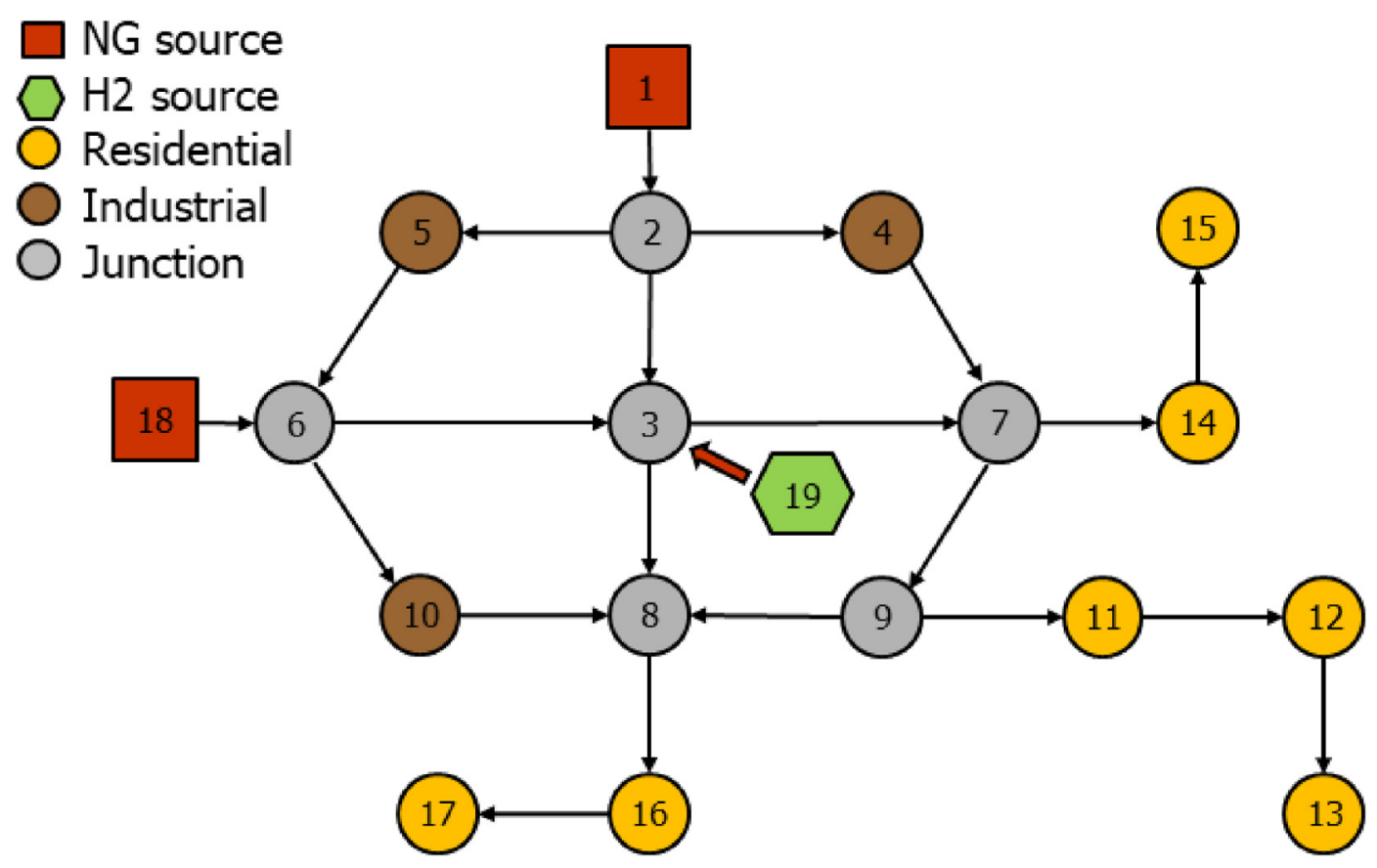

Fig. 1 - Natural gas grid layout.

typical winter day in climate zone " $\mathrm{D}$ " in the Italian region of Tuscany, the RES plants have been simulated [32,33] and the daily power production profile has been evaluated, as shown in Fig. 4.

The P.E.M. electrolyser considered has an estimated mean efficiency of $65 \%$ for the whole day and the production of green hydrogen has been estimated according to equation (1), where: $\eta_{e l}$ is the efficiency of the electrolyzer, $\mathrm{W}_{e l}$ is the power input and $\mathrm{LHV}_{\mathrm{H}_{2}}$ is the lower heating value for pure hydrogen [6].

$\dot{\mathrm{Q}}_{\mathrm{H}_{2}}=\frac{\eta_{e l} \mathrm{~W}_{e l}}{\mathrm{LHV}_{\mathrm{H}_{2}}}$

The electrolyser is used to produce green hydrogen when the electrical power produced by RES is bigger than the electrical power demand of the users. As can be seen from Fig. 5, hydrogen production is concentrated in the central hours of the day (10:00 to 13:00), corresponding to the peak of power production from PV panels, and in the evening hours, when the wind power produce reaches its peak and energy demand is reduced as industrial users switch off (20:00 to 24:00).

\section{Gas network steady-state model}

The gas distribution network is modelled through linear elements (pipes, valves and reducing stations), and point elements (junction nodes, which include interchange nodes and end various types of final users). The gas is injected into the distribution networks by specific elements called "City Gate Stations", which are the typical supply nodes of a gas distribution grid; due to their characteristics, pressure, temperature, and composition of the gas leaving the stations are usually known and set. Conversely, the mass flow rate is unknown because it depends on the gas requested by the downstream network layout. Interchange nodes are unconventional sources that, in injection mode, supply gas arriving from other networks or alternative gas (hydrogen, biogas/ biomethane, and synthetic natural gas) produced by renewable plants. The flow rate or the thermal power associated with the gas injected by interchange nodes into the grid is usually known and imposed as a boundary condition, while the injection pressure is a function of the conditions of the pipes connected to the considered node.

Boundary conditions and model approaches

Network users are connected to intermediate or final demand nodes and the gas extracted from these nodes depends on the consumption of users' devices. Consequently, the overall gas flow rate, required by the users, must be guaranteed simultaneously by the reduction cabins and by any interchange nodes or alternative gas injection points. Considering a constant composition of the gas flow, the higher heating value is also constant. Under these hypotheses, the flow rate ( $Q \cdot d m d$ )
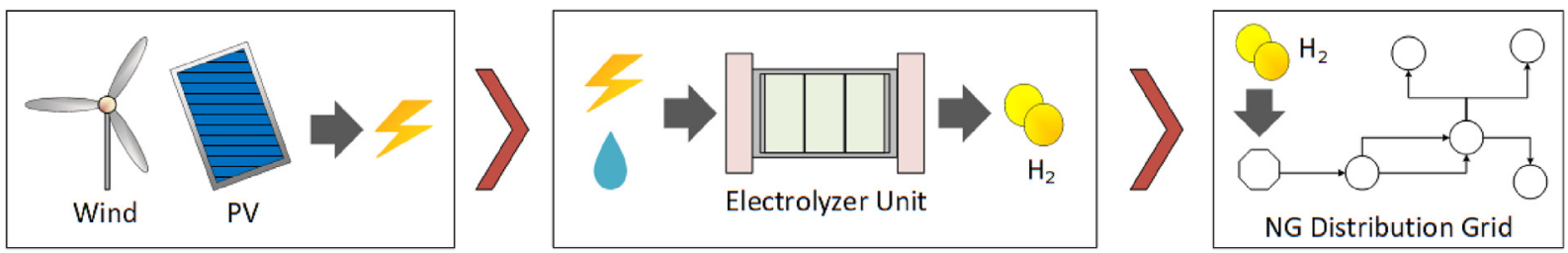

Fig. 2 - Power-to-gas system schematic diagram. 


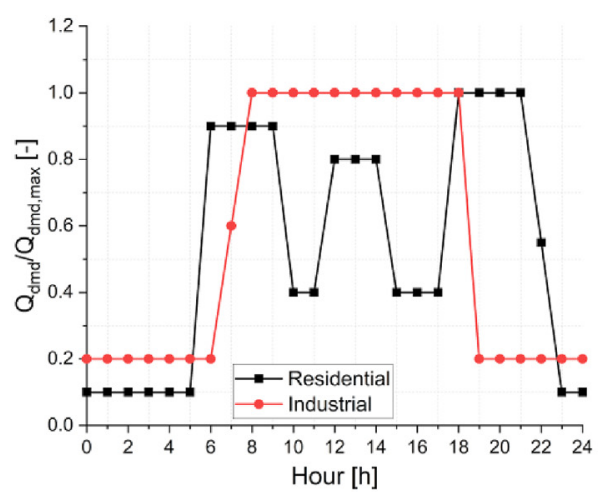

(a)

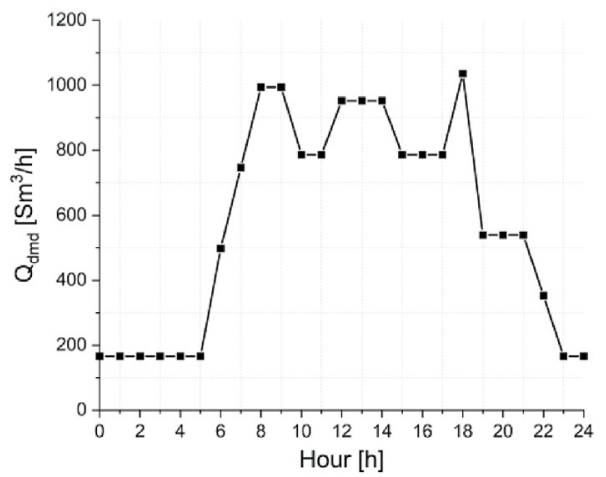

(c)

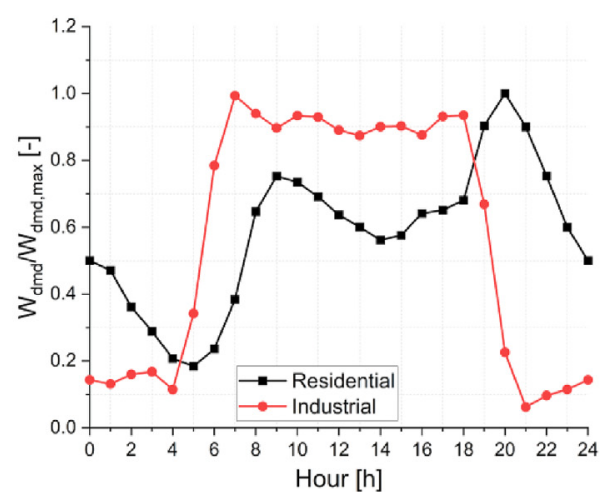

(b)

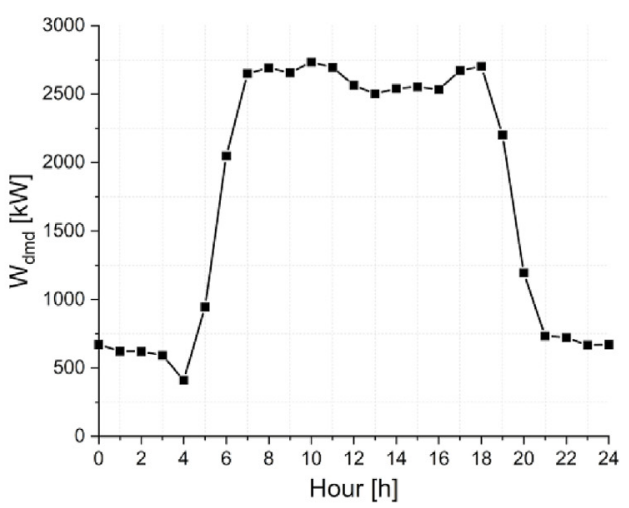

(d)

Fig. 3 - Dimensionless load profile for gas (a) and power (b) according to the considered users' classes. Total daily demand for gas (c) and power (d).

is proportional to the energy consumption and it can be set as a boundary condition. However, when gas composition and therefore higher heating value depend on the position of the demand node, the energy approach is more appropriate to describe the problem. The energy in unit of time requested by users (Wdmd) is set as a boundary condition, and the gas flow rate is calculated depending on the properties ( $\mathrm{HHV}$ ) of the local gas. Moreover, this approach becomes fundamental when starting to consider different types of gases injected into the gas network, as in the case of localized hydrogen injection.

$\dot{m}_{d m d}=\frac{\rho_{g} \dot{Q}_{d m d}}{3600}$

$\dot{m}_{d m d}=\frac{\rho_{g} W_{d m d}}{H H V_{g}}$

Equation of state and viscosity equation

To describe the natural gas or methane behaviour in a variable range of pressures is necessary to consider a compressibility factor $Z_{g}$ in the equation of state (4). The compressibility factor depends on the composition, temperature, and pressure of the gas mixture. The model used in the simulations implements the Papay [40] equation to evaluate the compressibility factor of gas mixture (6). The equation contains two dimensionless variables $T_{r}$ and $p_{r}$ which are respectively, the reduced temperature and the reduced pressures. These two variables are defined as the ratio between the value and the critical value of the properties.

$p_{g}=Z_{g} \rho_{g} R_{g} T_{g}$

$R_{g}=\frac{R_{u}}{\sum_{k=1}^{K} y_{k} M_{k}}$

$Z_{g}=1-3.52 p_{r} e^{-2.2660 T_{r}}+0.274 p_{r}^{2} e^{-1.878 T_{r}}$

$\mathrm{T}_{r}=\frac{\mathrm{T}_{g}}{\mathrm{~T}_{\mathrm{c}}}$

$p_{r}=\frac{p_{g}}{p_{c}}$

$\mathrm{T}_{\mathrm{c}}=\sum_{k=1}^{K} \frac{\mathrm{y}_{k} \mathrm{M}_{k}}{\mathrm{M}_{g}} \mathrm{~T}_{\mathrm{c}, \mathrm{k}}$ 


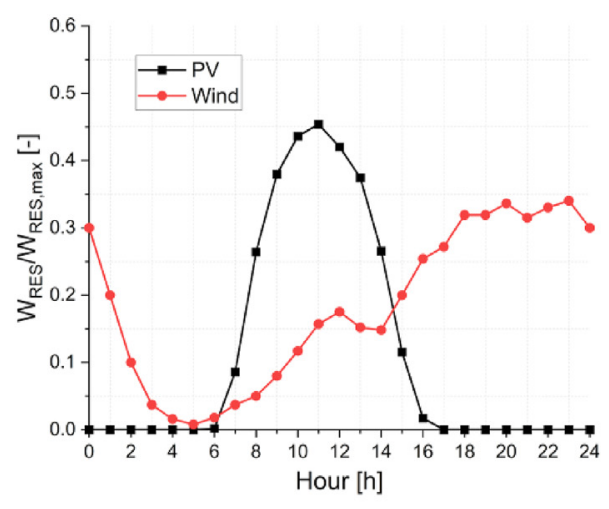

(a)

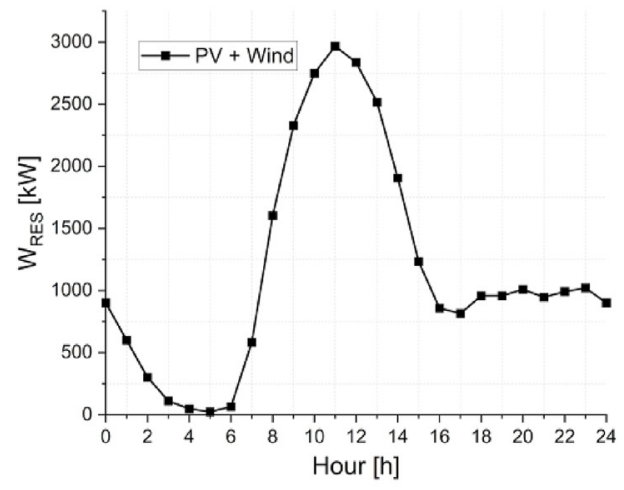

(b)

Fig. 4 - RES production dimensionless profiles for PV and Wind (a) and total RES production profile (b).

$p_{c}=\sum_{k=1}^{K} \frac{y_{k} M_{k}}{M_{g}} p_{c, k}$

To determine the gas flow regime and therefore the pressure losses occurring in the motion it is necessary to define how to evaluate the dynamic viscosity $\left(\mu_{g}\right)$ of a mixture. In a gas network, the dynamic viscosity depends on the composition of the gas mixture and the pressure and temperature conditions of the gas. Lucas method [41,42] has been implemented in the model used in this work to calculate the viscosity parameter $\left(\xi_{g}\right)$ and therefore the dynamic viscosity $\left(\mu_{g}\right)$ of a gas mixture (12). $F_{P}^{0}$ and $F_{Q}^{0}$ are two correction factors used to take into account polarity and quantum effects.

$\xi_{g}=0.176\left(\frac{T_{c}}{M_{g}^{3} P_{c}^{4}}\right)^{\frac{1}{6}}$

$\mu_{g}=\frac{\left(0.807 T_{r}^{0.618}-0.357^{-0.449 T_{r}}+0.340^{-4.058 T_{r}}+0.018\right) F_{P}^{0} F_{Q}^{0}}{\xi_{g}}$
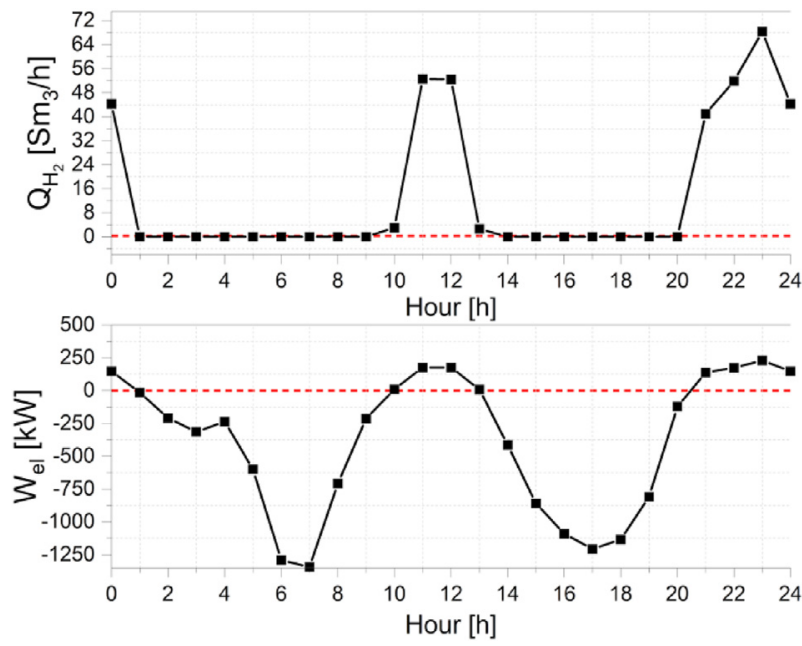

Fig. 5 - Hydrogen production for the considered reference day.

\section{Nodes model}

The node type elements can represent either gas injection points or gas passage and junction elements between two different pipes or end-users; The general nodes model provides $\mathrm{n}$ mass flow input, $\mathrm{m}$ mass flow output, and one mass flow demand. Mass flow rate, through inlet or outlet crosssection of the infinitesimal control volume, is proportional (13) to the gas density $(\rho)$, pipe cross-section area $(A)$, and velocity $(v)$.

In the model used for this work, the continuity stationary balance is applied to the overall gas flow rate (14) and to ensure the conservation of the mass flow of each of the $\mathrm{K}$ chemical species in the gas mixture (15).

$\dot{m}_{\mathrm{i}}=\rho \mathrm{Av}$

$\sum_{i=1}^{n} \dot{m}_{i, \text { in }}-\sum_{j=1}^{m} \dot{m}_{j, \text { out }}=\dot{m}_{d m d}$

$\sum_{i=1}^{n} \frac{y_{k} M_{k}}{M_{g}} \dot{m}_{i, i n}-\sum_{j=1}^{m} \frac{y_{k} M_{k}}{M_{g}} \dot{m}_{j, \text { out }}=\frac{y_{k} M_{k}}{M_{g}} \dot{m}_{d m d} \quad$ for $k=1, K$

Pipes model

The pipes of the gas network are classified according to the Italian standardization body (UNI), on the working pressure of the gas as high-pressure (species 1a, 2a, 3a), medium-pressure (species $4 a, 5 a, 6 a)$, and low-pressure (species 7a) pipes. Highpressure pipelines are used for long-range gas transport, while medium/low pressure pipelines are responsible for gas distribution in rural and urban areas. This article analyses a gas network made up of medium pressure pipes.

In the model presented pipes are linear elements crossed by a one-dimensional flow from an inlet to an outlet port. They are elements of the network where pressure losses of the gas occur. Distribution pipes are usually buried and therefore in this work is considered acceptable to impose isothermal condition and neglect heat exchange between the gas flow and pipe. The model also imposes a steady-state condition: a change of the outlet flow rate is immediately perceived at the inlet of the pipe. The model is based on the continuity equation and the momentum equations. 
Under the hypothesis above mentioned the continuity equation is translated in mass flow conservation for the gas mixture (16) and each of the $\mathrm{k}$ chemical species (17) composing the gas mixture, from inlet to outlet of a pipe.

$\dot{m}_{\text {in }}=\dot{m}_{\text {out }}$

$\frac{y_{k} M_{k}}{M_{g}} \dot{m}_{\text {in }}=\frac{y_{k} M_{k}}{M_{g}} \dot{m}_{\text {out }}$

The momentum equation relates the sum of the forces acting on the infinitesimal control volume to its rate of change of momentum. In gas networks where supply and demand nodes can have a different elevation of hundreds of meters, the pipe inclinations are not negligible because of the gravity effect. In this work, the Fergusson equation (18) has been chosen to model the momentum equations. The equation is valid under the assumption of a straight tube, one-dimensional steady flow, neglecting the gradient of velocity and imposing a constant density across each section of the tube [43].

$c_{1} p_{g, \text { in }}^{2}-p_{g, \text { out }}^{2}-c_{2} L|\dot{m}| \dot{m}=0$

$c_{1}=\exp \left(-c_{3}\right)$

$c_{2}=\frac{8 L}{\pi^{2} D^{5}} \lambda \underline{Z}_{g} T_{g} R_{g} \frac{\left(1-c_{1}\right)}{C_{3}}$

$c_{3}=\frac{2 g}{\underline{Z}_{g} T_{g} R_{g}} L \operatorname{sen} \theta$

$\underline{Z}_{g}=\frac{1}{L} \int_{x=0}^{x=L} Z_{g}(x) d x$

The coefficients that appear in the Fergusson equation are related to friction losses and the effects of gravity. When no elevation gain exists $\left(\theta=0^{\circ}\right)$, the coefficient $c 3$ is equal to zero, and the ratio $(1-c 1) / c 3$ becomes 1 . The coefficient $c 1$ assumes values bigger than 1 if the tube is ascending, smaller than 1 if the tube is descending and equal to 1 if the tube is flat. As the pressure changes along the length of the pipe, also the compressibility factor $(\mathrm{Zg})$ along the pipe is not constant. Therefore, the compressibility factor used in the Fergusson equation $(\bar{Z} g)$ is calculated, as the integral mean value of $(x)$ along the length $\mathrm{L}$ of the pipe (22).

For what concerns the friction forces the effects of internal friction between particles are neglected compared to the effect of pipe-gas friction. The friction factor $(\lambda)$ that appears in equation (20) is evaluated through equation (23) if the flow is laminar, while it is derived from the empirical DarcyWeisbach equation (24) if the flow is turbulent. The friction factor $(\lambda)$ is a function of roughness $(\varepsilon)$ and diameter $(D)$ of the pipe and regime of the fluid flow (Re) [44].

$\lambda=\frac{64}{\mathrm{Re}}($ Laminar Flow $)$

$\lambda=-2 \log \left(\frac{2.51}{\operatorname{Re} \sqrt{\lambda}}+\frac{1}{3.715} \frac{\varepsilon}{D}\right)$ (Turbulent Flow)

$\operatorname{Re}=\frac{\mathrm{D} \dot{m}}{\mathrm{~A} \mu_{g}}$

The present model does not include the energy equation because, as previously anticipated, the flow is considered at a constant temperature $(\mathrm{Tg}=\mathrm{cost})$, and the heat transfer between the flow and the pipe is neglected (see Table 2).

Gas quality model

Natural gas (NG) is a mixture of hydrocarbon gases extracted from underground reserves. The principal component of the mixture is methane $\left(\mathrm{CH}_{4}\right)$ and its mole fraction lies in a range between 80 and $99 \%$ of the mixture. The remaining hydrocarbon gases are ethane $\left(\mathrm{C}_{2} \mathrm{H}_{6}\right)$, propane $\left(\mathrm{C}_{3} \mathrm{H}_{8}\right)$, butane $\left(\mathrm{C}_{4} \mathrm{H}_{10}\right)$, pentane $\left(\mathrm{C}_{5} \mathrm{H}_{12}\right)$, and hexane $\left(\mathrm{C}_{6} \mathrm{H}_{14}\right)$. However, the mixture contains contaminant gas, such as carbon dioxide $\left(\mathrm{CO}_{2}\right)$, nitrogen $\left(\mathrm{N}_{2}\right)$, and helium $(\mathrm{He})$. The standard natural gas composition used in the gas grid simulations of this work is reported in Table 3.

Hydrogen (H2) can be injected into the gas network and mixed with natural gas. These injections influence the properties and quality of the gas delivered to users.

The most common indices used to monitor the gas quality are the Higher Heating Value (HHV), the specific gravity (SG), and the Wobbe Index (WI). The WI is a quantity derived by the HHV and the SG and therefore contains the information that belongs to both. The WI is used to analyse the interchangeability of two gas mixtures in terms of the heat rate supplied to the user.

$H H V_{g}=\sum_{k=1}^{n} y_{k} H H V_{k}$

$S G=\frac{\rho_{g, 0}}{\rho a i r, 0}$

$\mathrm{WI}=\frac{\mathrm{HHV}}{\sqrt{\mathrm{SG}}}$

The gas safety management regulations $[35,45]$ define the parameters range allowed to guarantee optimal combustion process of the devices connected to the grid (Table 4).

Table 3 - Standard natural gas composition vs Green Hydrogen composition.

$\mathrm{y}_{\mathrm{k}}[\%]$

\begin{tabular}{|c|c|c|c|c|c|c|c|c|c|c|}
\hline \multirow[b]{2}{*}{ Gas } & \\
\hline & $\mathrm{CH}_{4}$ & $\mathrm{C}_{2} \mathrm{H}_{6}$ & $\mathrm{C}_{3} \mathrm{H}_{8}$ & $\mathrm{C}_{4} \mathrm{H}_{10}$ & $\mathrm{C}_{5} \mathrm{H}_{12}$ & $\mathrm{C}_{6} \mathrm{H}_{14}$ & $\mathrm{CO}_{2}$ & $\mathrm{~N}_{2}$ & $\mathrm{He}$ & $\mathrm{H}_{2}$ \\
\hline Standard NG & 97.201 & 1.862 & 0.393 & - & - & - & - & 0.544 & - & - \\
\hline Green Hydrogen & - & - & - & - & - & - & - & - & - & 100.0 \\
\hline
\end{tabular}


Table 4 - Gas lower and higher limits for the three main performance indexes.

\begin{tabular}{lcc} 
Parameter & $\begin{array}{c}\text { Lower Limit } \\
\text { Value }\end{array}$ & $\begin{array}{c}\text { Higher Limit } \\
\text { Value }\end{array}$ \\
\hline Wobbe Index [MJ/Sm ${ }^{3}$ ] & 47.2 & 52.2 \\
Higher Heating Value $\left[\mathrm{MJ} / \mathrm{Sm}^{3}\right]$ & 34.95 & 45.28 \\
Specific Gravity [-] & 0.55 & 0.7 \\
\hline
\end{tabular}

\section{Results}

In this section the results of the case study simulations are presented:

- An investigation under nominal condition (single hour gas demand according to Table 1) compares the gas grid behaviour using only natural gas with the case of a percentage of hydrogen injected in the total flow: two boundary-conditions approaches (total gas flow demand or total energy demand) are discussed.

- The same grid is simulated over $24 \mathrm{~h}$ in "energy" mode imposing the gas profile requested by the users as a boundary condition; the profile of hydrogen generated from the RES surplus is used as input for the analysis. Two control logics for the injection are proposed: limiting the molar percentage of hydrogen injected to the $10 \%$, referred to the total gas demand of the grid or injecting a constant amount of hydrogen equal to the daily average production. The first control logic is used to carry out a sensitivity analysis on the node where hydrogen is injected.

The results highlight the effect of hydrogen injection on quality indexes and fluid-dynamic parameters of the gas flow. A special focus is given to the Wobbe index whose values, in the energy mode analysis, are associated with a chromatic scale and presented on the map of the grid (see Table 4).

\section{Nominal conditions analysis}

Hydrogen injections analysis approaches

Table 5 shows the results of the simulation of the natural gas network in nominal reference conditions without hydrogen injection; the values of these parameters are used to weight the results obtained in the simulation of

\section{Table 5 - Reference case results.}

\begin{tabular}{lll} 
Index & Units & Value \\
\hline $\mathrm{WI}_{\min }$ & $\mathrm{MJ} / \mathrm{Sm}^{3}$ & 50.74186 \\
$\mathrm{HHV}_{\text {min }}$ & $\mathrm{MJ} / \mathrm{Sm}^{3}$ & 38.27491 \\
$\mathrm{SG}$ & - & 0.568978 \\
$\mathrm{H}_{2}$ & $\%$ & 0 \\
$\mathrm{Q}_{\text {tot }}$ & $\mathrm{Sm} / \mathrm{h}$ & 1035 \\
$\mathrm{~W}_{\text {tot }}$ & $\mathrm{MW}$ & 11 \\
$\mathrm{P}_{\min }$ & $\mathrm{bar}$ & 0.483636 \\
$\mathrm{~V}_{\max }$ & $\mathrm{m} / \mathrm{s}$ & 5.9312 \\
$\rho$ & $\mathrm{kg} / \mathrm{Sm}^{3}$ & 0.98 \\
Gas ID & - & $\mathrm{SNG}$ \\
\hline
\end{tabular}

the grid with hydrogen injection to give an effective comparison.

As previously described in section Boundary conditions and model approaches, the system of equations describing the grid can be solved by imposing, as a boundary condition, the gas flow rate or the energy required by the users.

The comparison in Fig. 6 has been carried out by injecting at node 3 , which is the junction closest to the CGS nodes, a quantity of hydrogen equal to $600 \mathrm{~kJ} / \mathrm{s}$, which corresponds to a hydrogen volumetric flow of about $178.7 \mathrm{Sm}^{3} / \mathrm{h}$. The flow method (pink) keeps the gas flow constant (equal to $Q_{\text {tot }}$ in Table 3) which implies lower total energy of the network due to the injection of a gas with a lower volumetric calorific value. The energy method, respect to the flow method, keeps the required energy constant (equal to $\mathrm{W}_{\text {tot }}$ in Table 3 ) by increasing the flow of natural gas required by the users. In both cases, it is possible to appreciate a reduction of quality parameters such as SG, HHV, and consequently WI due to the characteristics of hydrogen. The energy approach, which is conceptually the right one, results more critical for the pipelines as the maximum velocities in the pipes and therefore the pressure losses increase. The Wobbe Index values obtained in energy mode are associated with a chromatic scale, helping to understand which junctions are affected by the hydrogen injection. In Fig. 7 is possible to appreciate the reduction of the WI downstream the injection node. The WI reaches values close to the $\mathrm{WI}_{\min }$ suggested by the Italian regulatory authority. Node 10 has a lower WI index because it is an extraction point whose gas is the result of a mixture from two different streams from junction 6 and junction 8 .

\section{Pattern analysis}

Single node injection

Fig. 8 shows the results of the network analysis hour by hour when the green hydrogen is injected in correspondence of node 3 according to the coupling between the RES hourly production and the users' hourly total demand; Fig. 8a shows the daily pattern of hydrogen volume injection according to

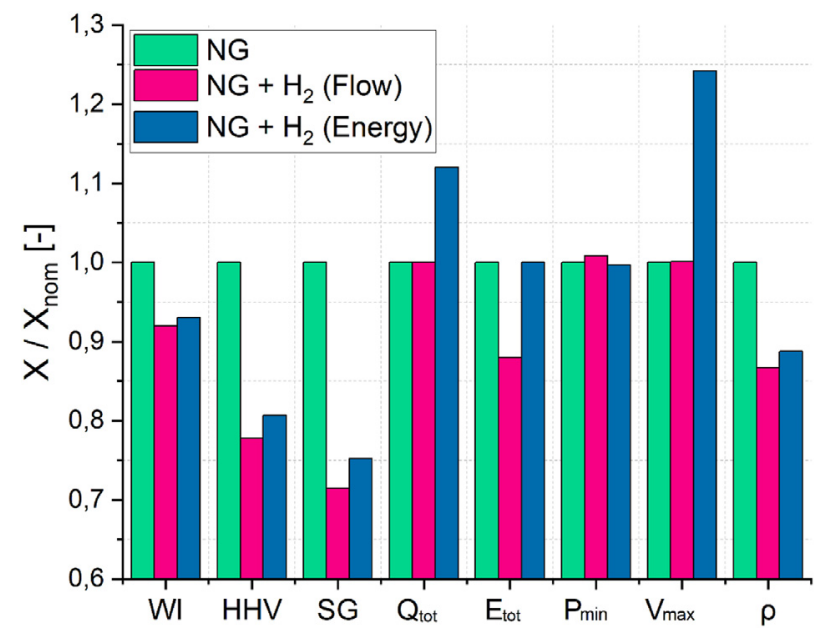

Fig. 6 - Reference case results comparison: only NG vs $\mathrm{H} 2$ (Flow \& Energy Method). 


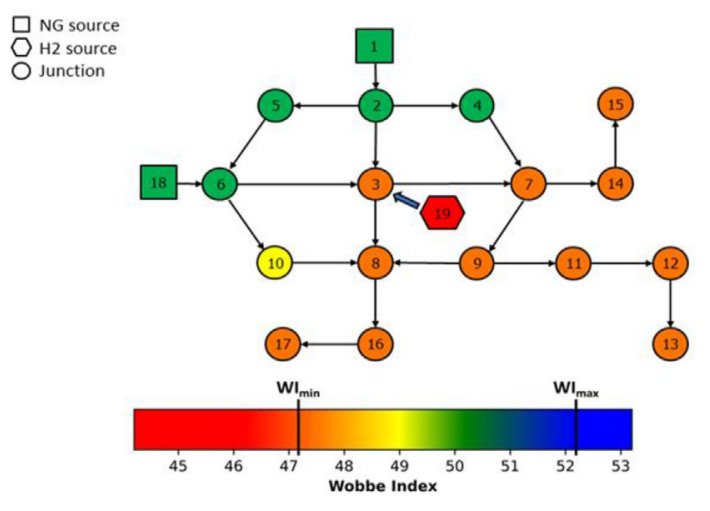

(a)

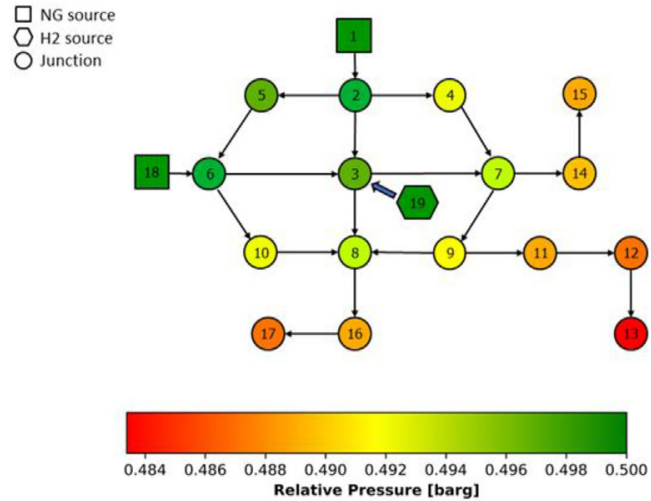

(b)

Fig. 7 - Wobbe Index and pressure values' variation map under nominal conditions.

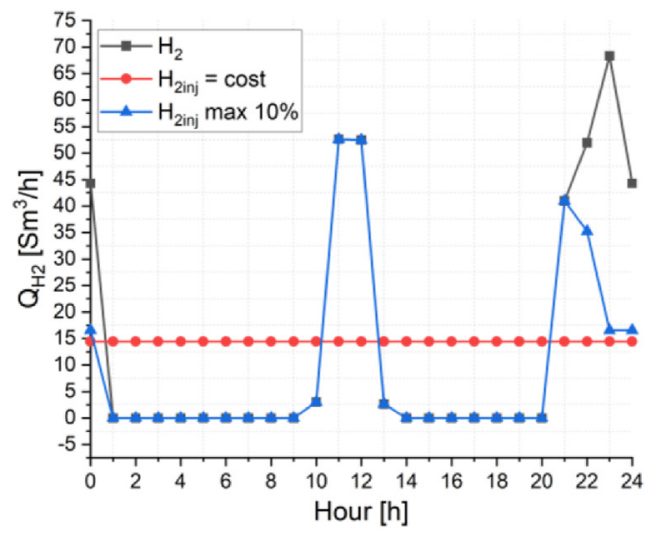

(a)

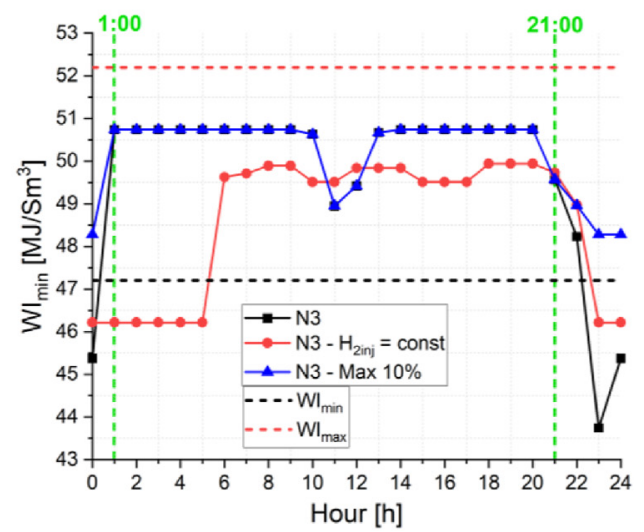

(b)

Fig. 8 - (a) Hydrogen injection patterns; (b) Pattern analysis: Node 3 injection, network minimum Wobbe Index during the day without control logics (black) vs with control logics (red, blue). (For interpretation of the references to color in this figure legend, the reader is referred to the Web version of this article.)

the considered logic, while Fig. $8 \mathrm{~b}$ presents the minimum WI values during the day. The three lines represent the minimum WI reached in the grid during the day considering two different injection scenarios: injecting all the hydrogen produced by the surplus of power coming from RES (black line), injecting hydrogen with a control logic which limits during the night (from 21:00 to 01:00) the maximum molar percentage of hydrogen injected in the flow to $10 \%$ (blue line) and injecting a constant flow of hydrogen equal to the mean value of the total amount of hydrogen produced by the RES in the considered day (red line). Focusing on the black line, two valleys in the profile of the network's WI minimum value are shown: the first is about $48.9 \mathrm{MJ} / \mathrm{Sm} 3$ at 11:00 in correspondence of the PV production peak and the second is about $43.5 \mathrm{MJ} / \mathrm{Sm} 3$ at 23:00, when the wind production reaches the maximum and the users' demand is lower because of the industries shut down. The value reached by the WI at 23:00 is largely smaller than the $\mathrm{WI}_{\text {min }}$ admitted. Control logic is necessary to ensure that the power from RES converted in green hydrogen and injected into the grid does not lead the WI to overcome the lower threshold. The control logics hide a scenario where a percentage of the power produced from RES must be stored in a battery, or hydrogen must be stored in a tank increasing the costs of the system.

The comparison between the red and the blue line shows that both control strategies ensure the Wobbe Index to stay in the safety band, but its evolution during the day is different. Injecting a constant amount of hydrogen implies that the minimum of the Wobbe Index is reached during the night where the gas demand is lower for a longer period (from 23:00 to $05: 00$ ). The WI profile during the day is characterized by just one valley during the night. The lunch valley disappears because with this control strategy, once the constant amount of hydrogen injected is calculated, the monotonicity intervals of the WI profile are a function of the gas demand profile and during lunch, it is constant as shown in Fig. 3.

Injection nodes sensitivity analysis

The reference gas network was previously studied by Adolfo and Carcasci [28], which carried on a sensitivity analysis of the gas network behaviour while changing the injection point: three clusters of different behaviour were found.

The sensitivity analysis carried out by Adolfo and Carcasci highlights that the more upstream you inject hydrogen in the 


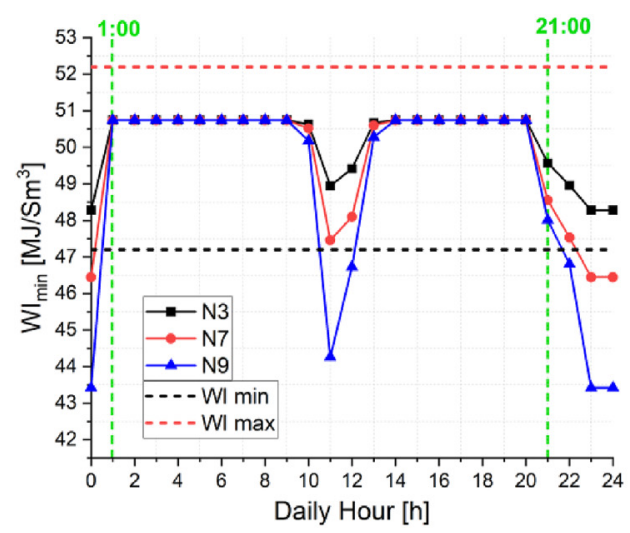

(a)

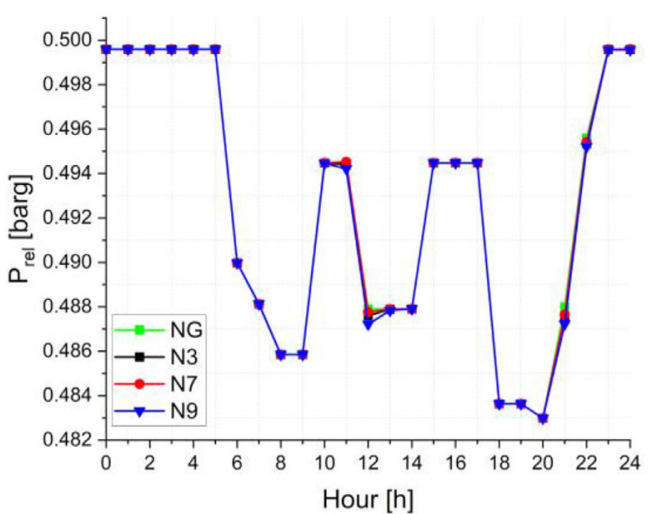

(b)

Fig. 9 - Injection position sensitivity analysis (a) and Relative pressure pattern analysis (b).

grid and the less the minimum Wobbe Index of the grid will decrease. Anyway, the choice of the injection node depends also on geographical restriction (e.g. where the RES are and where the elctrolyser is).

The results of this work are obtained using N3, N7, and N9 as injection points for the green hydrogen and considering a control logic that limits the hydrogen percentage to the $10 \%$ in volume during the night. N3 is a central node and all the user nodes (except node 5) are reached by the flow exiting N3. N7 is a node that underlies industrial (N4) and residential user (N14, N15). N9 is a node that underlies residential users only (N11, N12, N13). Focusing on the minimum value reached by the Wobbe Index in the grid (Fig. 9a), the injection at node 9 is still the most penalizing solution while the injection at node 3 is the best solution for coupling the reference gas grid to the renewable production. Focusing on the PV peak hour, i.e., $11: 00$, the minimum WI is reduced by $3.54 \%, 6.46 \%$ and $12.77 \%$ of the nominal value, while at the $23: 00$, in correspondence of the wind production peak, we found $4.85 \%, 8.46 \%, 14.43 \%$ reduction of the minimum WI. Fig. $9 \mathrm{~b}$ highlights three aspects: i) each hydrogen's injection position implies a reduction, in comparison to $100 \%$ NG grid, of the minimum pressure in the grid, ii) the differences regarding the minimum pressure in the grid are remarkable only around the peak of hydrogen injection (11:00 to $12: 00$ and $21: 00$ to $22: 00$ ), iii) injecting in correspondence of node 9 returns always the lowest values of minimum pressure during the day. Anyway, even if the injection of hydrogen leads to a reduction of the pressure of the flow, the values obtained are largely into the safety range imposed by ARERA for NG flowing in the 6th species pipes. Therefore, another conclusion that can be obtained by the results in Fig. 9 is that WI represents the most restrictive condition to be achieved.

In Fig. 10 a comparison of the WI values obtained by the simulation injecting hydrogen in nodes 3, 7 and 9 at 11:00 is presented. Each junction has been coloured according to the same WI chromatic scale, which was also used in Fig. 8. Both the injection in node 3 and node 7 ensure to respect the WI's safety limits but node 3 ensures a larger margin thanks to the larger number of users reached by hydrogen. Injecting
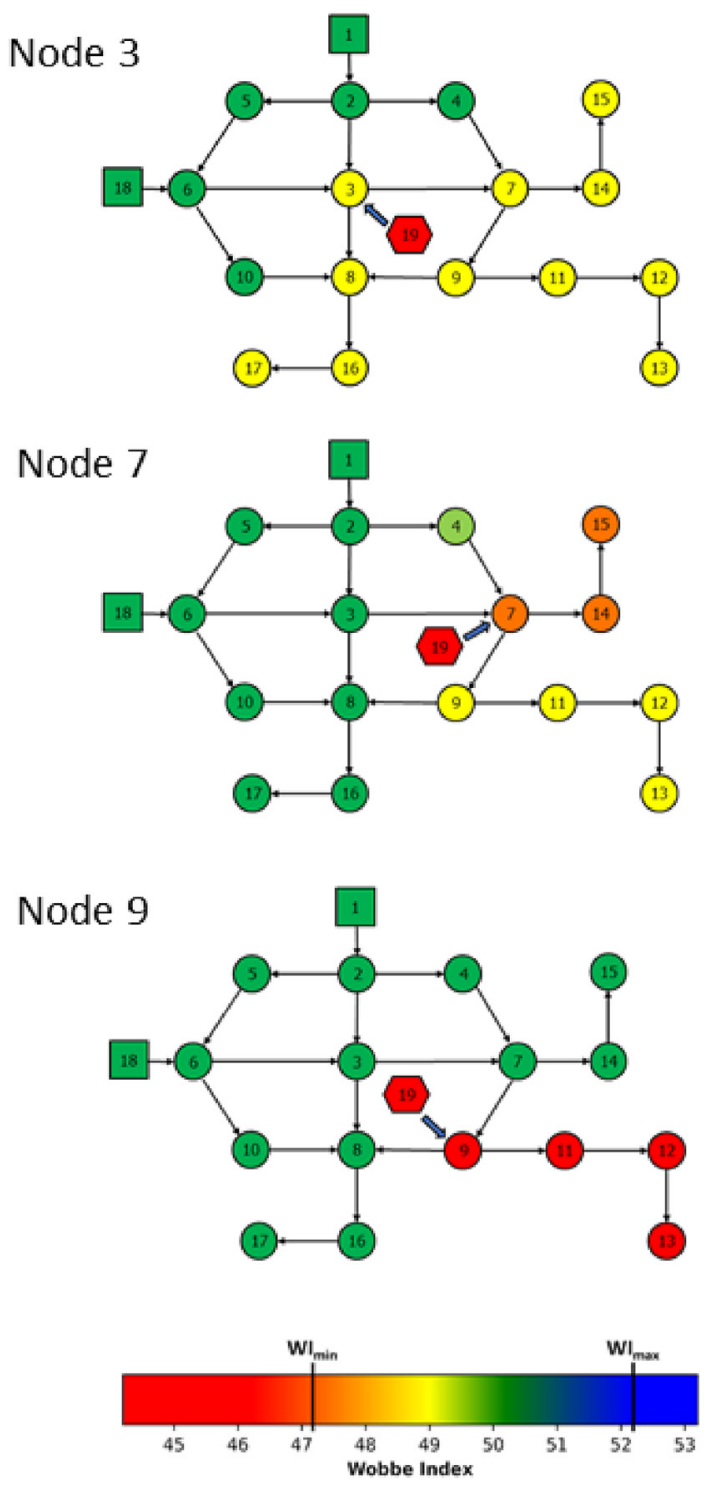

Fig. 10 - Wobbe Index variation maps: comparison of the effects of three different injection's nodes. 


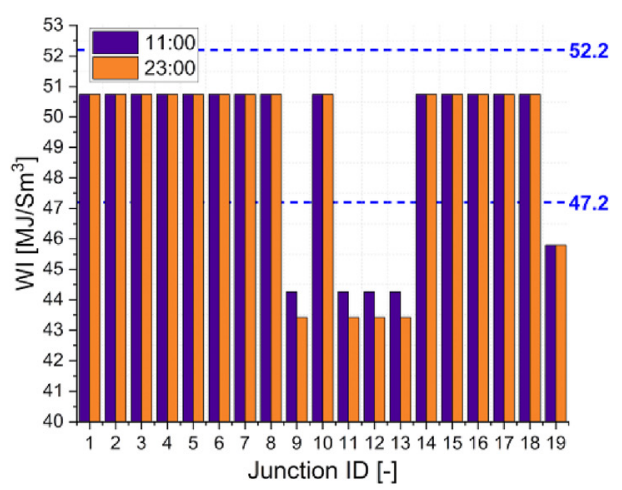

(a)

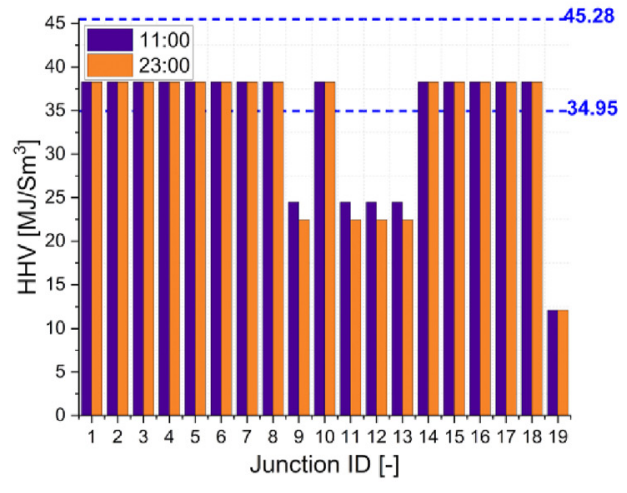

(c)

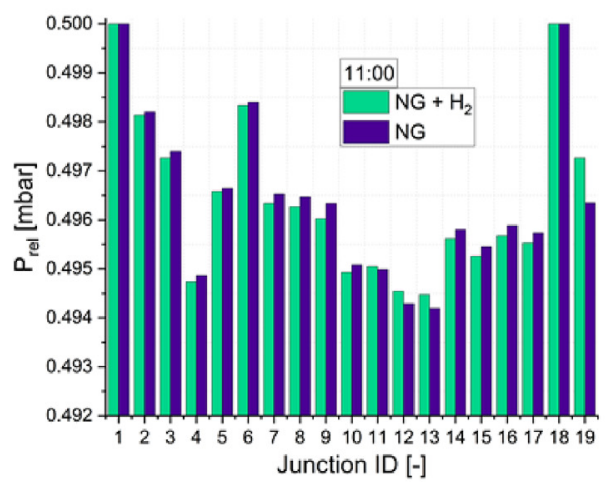

(e)

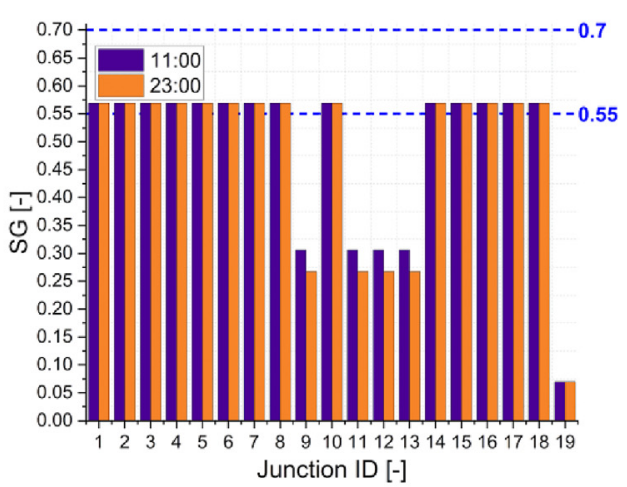

(b)

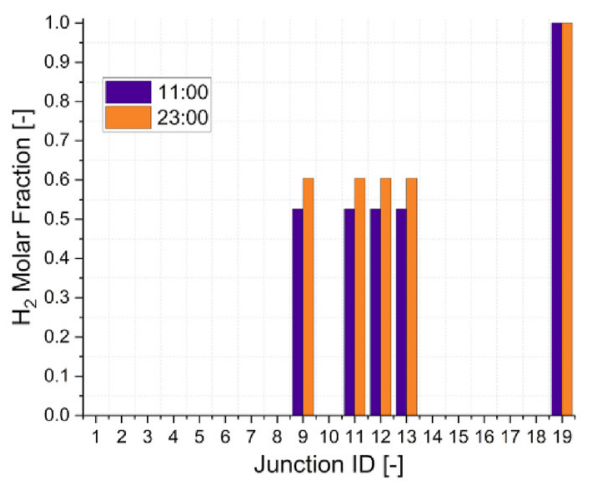

(d)

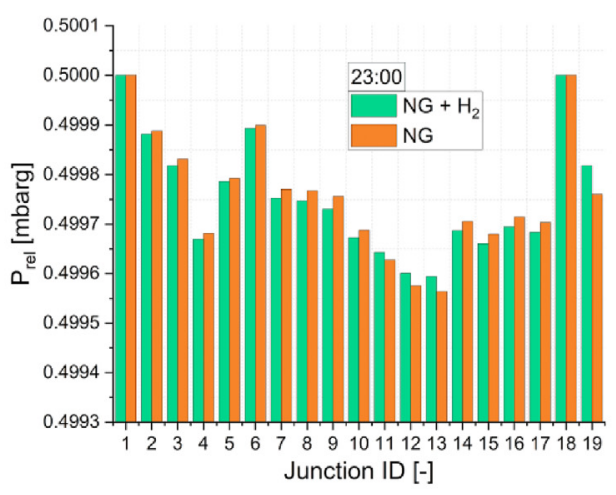

(f)

Fig. 11 - N9 injection case at 11:00 and 23:00. Junctions WI (a), SG (b), HHV (c), H2 molar fraction (d).Junctions relative pressures $(e-f)$ values compared to the nominal case with NG only.

hydrogen in node 9 brings all the downstream nodes to overcome the lower limit of WI admitted not ensuring the safety of the users 11,12 , and 13.

Junctions analysis in case of single node hydrogen injection In this paragraph, the behaviour of the grid is presented for the case of injection in node 9. The injection follows the control logic that limits the hydrogen's molar percentage to $10 \%$ of the users' total demand of the grid.
The results are presented for two reference daily hours: 11:00 and 23:00 corresponding to the PV and wind peak of power production, respectively.

The nodes from 11 to 13 represent the residential users located downstream of the injection node 9 at the end of the considered gas network. The mentioned nodes are affected by the presence of hydrogen in the final delivered gas mixture. Fig. 11d shows that the molar fraction of the injected hydrogen is about $50 \%$ at 11:00, while at 23:00 the 


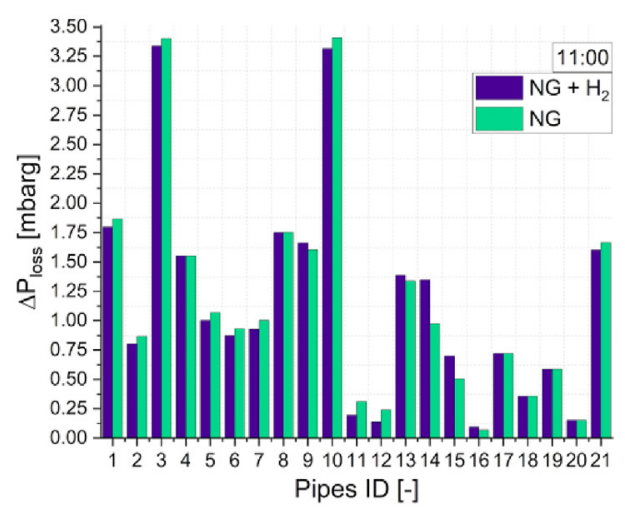

(a)

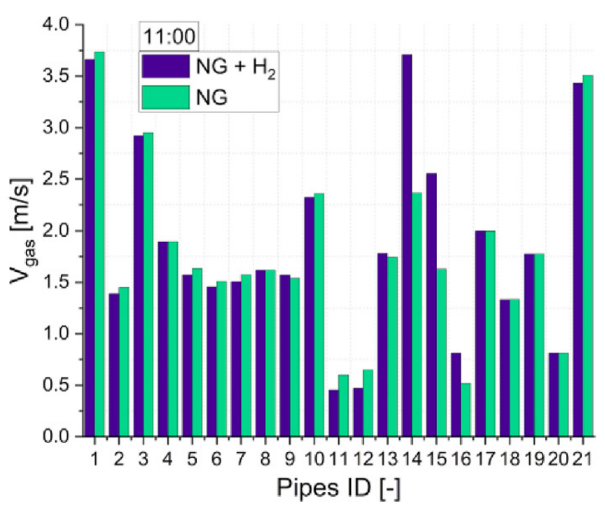

(c)

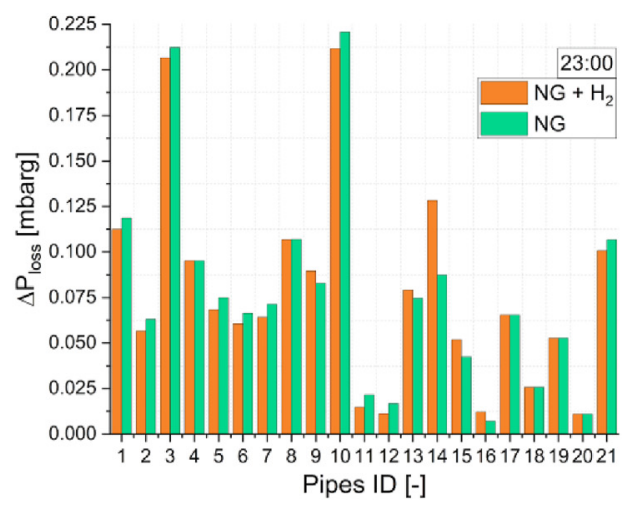

(b)

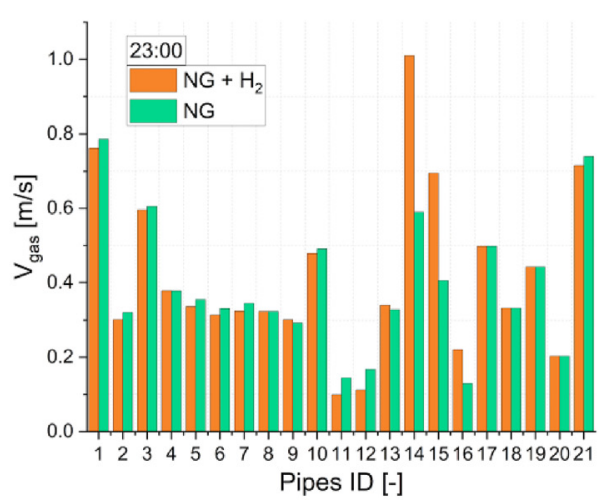

(d)

Fig. 12 - Pipes pressure losses at 11.00 (a) and at 23:00 (b) compared to the nominal case with NG only. Max gas velocity at 11.00 (c) and at 23:00 (d) compared to the nominal case with NG only.

maximum molar fraction for the mentioned users reaches the value of about $60 \%$ of the gas mixture. Fig. $11 \mathrm{a}, \mathrm{b}$, and 11c show the WI, SG, and HHV node by node: the lower limits of the considered performance indexes are breached both at 11:00 and 23:00. In Fig. 11e and $\mathrm{f}$ it is possible to see the pressure returned by the simulations (NG only and hydrogen blended in NG) in each junction. The results obtained show the grid having the same behaviour at different times of the day. The main conclusion derived from Fig. 11 is that the behaviour of the junctions is different according to their position in the grid. The nodes characterized by the same pressures in the comparison (node 1 and node 18) are the NG sources. The nodes characterized by higher pressure in the hydrogen injection case are the nodes located upstream of the hydrogen injection node. This behaviour is justified considering that the inlet pressure of NG from the sources is the same but the flow and therefore the losses in the pipes are lower as part of the flow is supplied by the hydrogen injection downstream. The nodes characterized by lower pressure in the hydrogen injection case are located downstream of the hydrogen injection node (node 9). The last consideration deserves a deepening: while a node located upstream of the hydrogen injection point will always have a higher pressure, a node located downstream may have a lower or a higher pressure compared to the NG case. The hydrogen injection node (node 9) will always have a higher pressure if compared to the NG case but the flow and therefore the pressure losses in the pipes downstream that node is higher (higher flow is needed if hydrogen is used to supply a certain energy demand). Focusing on the grid proposed in this work, the increment of pressure in node 9 is lower than the losses obtained in the pipe that links node 9 to node 11 and therefore node 11 is characterized by lower pressure in the hydrogen injection case. The same conclusion can be derived for the nodes 12 and 13 located downstream.

Pipes analysis in case of single node hydrogen injection In this section are presented the pressure losses and velocity of the gas across each pipe. Following the model implemented, injecting hydrogen reduces the HHV of the mixture and forces the volumetric flow to increase to keep fixed the energy supplied to the users. As the volumetric flow increases the velocity increases too. As the friction term of (18) is proportional to the velocity, in the pipes where the hydrogen flows the velocity is higher and the pressure losses are higher 
if compared to the case of $100 \%$ natural gas flowing in the same pipes. The previous considerations are shown in Fig. 12 for what concerns pipes 13 to 16. Instead, as the injection of hydrogen is at node 9 , less natural gas must be supplied by the CGS. A lower mass flow in the pipes upstream node 9 is reflected in a lower velocity of the flow in the pipes and, as the density is constant, also in lower pressure losses. The pipes in which the velocity and the pressure losses appear to be constant, comparing the two simulations, are the ones in which the natural gas flowing will not be mixed with hydrogen downstream.

\section{Conclusions}

The presented work aims to recreate a small, simplified, and representative urban gas network to investigate the effects of injecting green hydrogen produced from local renewable sources. The case study is composed of two elements: a natural gas distribution network to which clusters of residential and industrial users are connected and a power-to-gas system, composed of one photovoltaic field and one wind farm coupled with a P.E.M. electrolyser to produce green hydrogen. A steady-state model was developed to analyse a gas network in the presence of distributed hydrogen injection. Initially, the gas grid is analysed under nominal conditions of gas flow demand, and two approaches are compared for the injection of the same amount of hydrogen gas; the energy method, which imposes the energy to be delivered to the users, increasing the gas flow, was chosen as the more suitable for the successive simulations. Afterwards, a typical winter day was simulated: the production from renewable energy sources and the users' demands were simulated for a region of central Italy and the hourly power surplus was transferred to an electrolyser to produce green hydrogen to be injected into the network.

The results show that the injection of hydrogen into a local gas grid mainly affects the Wobbe index which highly decreases when hydrogen gas is injected into the network. The most critical situation during a typical winter day in Tuscany is shown to be at 11:00 and 23:00 in correspondence with the peaks of power surplus generated by RES. A comparative analysis shows the response of the grid changing three different injection nodes. The standard quality parameters are respected by injecting the hydrogen produced by the surplus of power in node 3 only if a control algorithm is applied. The effects of two control algorithms were investigated. The first one limits the maximum amount of the molar fraction of hydrogen to $10 \%$ of the total gas demand and creates two local minimums in the Wobbe Index daily variation, while injecting a constant amount of hydrogen shows a single but longer minimum during night-time. The first control algorithm presented ensures to respect the safety range of Wobbe index admitted if the injection of hydrogen was in node 3. A change of the hydrogen injection point to node 7 and node 9 leads to overcoming the minimum Wobbe Index and not ensuring safety standards to the users. To overcome this problem, a dedicated control logic must be realized for different nodes. The last part of the paper focuses on a comparison between the $100 \%$ NG simulation and the simulation considering a hydrogen injection in the most critical node (node 9). The gas quality parameters (HHV, SG, WI) are constant upstream while decrease downstream of the hydrogen injection node (exceeding the range of values admitted). It is also shown that pressure in the junctions upstream the hydrogen injection node increases because velocity and pressure losses in the upstream pipes decrease. Instead, the pressure in the junctions downstream the hydrogen injection node decreases because velocity and pressure losses in the downstream pipes increase.

The gas network chosen as a case study appears suitable to absorb the surplus of electricity generated by RES, but the choice of the hydrogen injection node, as well as the control strategy, results critical for the maximum amount of injectable hydrogen.

\section{Declaration of competing interest}

The authors declare that they have no known competing financial interests or personal relationships that could have appeared to influence the work reported in this paper.

\section{REFERENCES}

[1] Pellegrino S, Lanzini A, Leone e P. «Greening the gas network - the need for modelling the distributed injection of alternative fuels». Renew Sustain Energy Rev 2017;70:266-86. https://doi.org/10.1016/j.rser.2016.11.243. apr.

[2] ENTSOG Roadmap 2050. ENTSOG, https://www.entsog.eu/ entsog-roadmap-2050.

[3] Gas decarbonisation pathways study. Gas for Climate 2050 2020;(7):108-26. https://gasforclimate2050.eu/sdm _ downloads/2020-gas-decarbonisation-pathways-study/.

[4] HE Vision on the Role of Hydrogen and Gas Infrastructure on the Road Toward a Climate Neutral Economy - A Contribution to the Transition of the Gas Market | Hydro». https:// hydrogeneurope.eu/news/he-vision-role-hydrogen-and-gasinfrastructure-road-toward-climate-neutral-economy. Chapter 3: 7-13.

[5] Gondal IA. Hydrogen integration in power-to-gas networks. Int J Hydrogen Energy 2019;44(3):1803-15. https://doi.org/ 10.1016/j.ijhydene.2018.11.164. gen.

[6] Buttler A, Spliethoff H. Current status of water electrolysis for energy storage, grid balancing and sector coupling via power-to-gas and power-to-liquids: a review. Renew Sustain Energy Rev 2018;82:2440-54. https://doi.org/10.1016/ j.rser.2017.09.003. feb.

[7] Gahleitner G. Hydrogen from renewable electricity: an international review of power-to-gas pilot plants for stationary applications. Int J Hydrogen Energy 2013;38(5):2039-61. https://doi.org/10.1016/ j.ijhydene.2012.12.010. feb.

[8] Qadrdan M, Abeysekera M, Chaudry M, Wu J, Jenkins e N. Role of power-to-gas in an integrated gas and electricity system in Great Britain. Int J Hydrogen Energy 2015;40(17):5763-75. https://doi.org/10.1016/ j.ijhydene.2015.03.004. mag.

[9] Zachariah-Wolff JL, Egyedi TM, Hemmes e K. From natural gas to hydrogen via the Wobbe index: the role of standardized gateways in sustainable infrastructure transitions. Int J Hydrogen Energy 2007;32(9):1235-45. https://doi.org/10.1016/j.ijhydene.2006.07.024. giu. 
[10] Estermann T, Newborough M, Sterner e M. Power-to-gas systems for absorbing excess solar power in electricity distribution networks. Int J Hydrogen Energy 2016;41(32):13950-9. https://doi.org/10.1016/ j.ijhydene.2016.05.278. ago.

[11] Quarton CJ, Samsatli S. Power-to-gas for injection into the gas grid: what can we learn from real-life projects, economic assessments and systems modelling? Renew Sustain Energy Rev 2018;98:302-16. https://doi.org/10.1016/ j.rser.2018.09.007. dic.

[12] Guandalini G, Campanari S. Wind power plant and power-togas system coupled with natural gas grid infrastructure: techno-economic optimization of operation. In: Oil and gas applications; supercritical $\mathrm{CO} 2$ power cycles; wind energy, montreal, quebec, Canada, vol. 9; 2015, V009T46A004. https:// doi.org/10.1115/GT2015-42229. giu.

[13] Fischer D, Kaufmann F, Selinger-Lutz O, Voglstätter e C. Power-to-gas in a smart city context - influence of network restrictions and possible solutions using on-site storage and model predictive controls. Int J Hydrogen Energy 2018;43(20):9483-94. https://doi.org/10.1016/ j.ijhydene.2018.04.034. mag.

[14] Wang B, Liang Y, Zheng J, Qiu R, Yuan M, Zhang e H. An MILP model for the reformation of natural gas pipeline networks with hydrogen injection. Int J Hydrogen Energy 2018;43(33):16141-53. https://doi.org/10.1016/ j.ijhydene.2018.06.161. ago.

[15] Witkowski A, Rusin A, Majkut M, Stolecka e K. Analysis of compression and transport of the methane/hydrogen mixture in existing natural gas pipelines. Int J Pres Ves Pip 2018;166:24-34. https://doi.org/10.1016/j.ijpvp.2018.08.002. set.

[16] Weber AC, Papageorgiou LG. Design of hydrogen transmission pipeline networks with hydraulics. Chem Eng Res Des 2018;131:266-78. https://doi.org/10.1016/ j.cherd.2018.01.022. mar.

[17] He G-X, Yan H, Chen L, Tao e W-Q. Economic dispatch analysis of regional Electricity-Gas system integrated with distributed gas injection. Energy 2020;201:117512. https:// doi.org/10.1016/j.energy.2020.117512. giu.

[18] labidine Messaoudani Z, Rigas F, Binti Hamid MD, Che Hassan e CR. Hazards, safety and knowledge gaps on hydrogen transmission via natural gas grid: a critical review. Int J Hydrogen Energy 2016;41(39):17511-25. https://doi.org/ 10.1016/j.ijhydene.2016.07.171. ott.

[19] Abd AA, Naji SZ, Thian TC, Othman e MR. Evaluation of hydrogen concentration effect on the natural gas properties and flow performance. Int J Hydrogen Energy 2021;46(1):974-83. https://doi.org/10.1016/ j.ijhydene.2020.09.141. gen.

[20] Bouledroua O, Hafsi Z, Djukic MB, Elaoud e S. The synergistic effects of hydrogen embrittlement and transient gas flow conditions on integrity assessment of a precracked steel pipeline. Int J Hydrogen Energy 2020;45(35):18010-20. https:// doi.org/10.1016/j.ijhydene.2020.04.262. lug.

[21] Zhao Y, McDonell V, Samuelsen e S. Influence of hydrogen addition to pipeline natural gas on the combustion performance of a cooktop burner. Int J Hydrogen Energy 2019;44(23):12239-53. https://doi.org/10.1016/ j.ijhydene.2019.03.100. mag.

[22] Simonis B, Newborough M. Sizing and operating power-togas systems to absorb excess renewable electricity. Int J Hydrogen Energy 2017;42(34):21635-47. https://doi.org/ 10.1016/j.ijhydene.2017.07.121. ago.

[23] Adolfo D, Carcasci C. Unsteady simulation of natural gas networks. India: Erode; 2019. p. 20001. https://doi.org/ 10.1063/1.5138734.
[24] Mazza A, Cavana M, Mercado Medina EL, Chicco G, Leone P. Creation of representative gas distribution networks for multi-vector energy system studies. In: 2019 IEEE international conference on environment and electrical engineering and 2019 IEEE industrial and commercial power systems Europe (EEEIC/I\&CPS Europe), genova, Italy; 2019. p. 1-6. https://doi.org/10.1109/EEEIC.2019.8783701. giu.

[25] Guandalini G, Colbertaldo P, Campanari e S. Dynamic modeling of natural gas quality within transport pipelines in presence of hydrogen injections. Appl Energy 2017;185:1712-23. https://doi.org/10.1016/ j.apenergy.2016.03.006. gen.

[26] Chaczykowski M, Sund F, Zarodkiewicz P, Hope e SM. Gas composition tracking in transient pipeline flow. J Nat Gas Sci Eng 2018;55:321-30. https://doi.org/10.1016/ j.jngse.2018.03.014. lug.

[27] Abeysekera M, Wu J, Jenkins N, Rees e M. Steady state analysis of gas networks with distributed injection of alternative gas. Appl Energy 2016;164:991-1002. https:// doi.org/10.1016/j.apenergy.2015.05.099. feb.

[28] Abeysekera M, Rees M, Wu e J. Simulation and analysis of low pressure gas networks with decentralized fuel injection. Energy Procedia 2014;61:402-6. https://doi.org/10.1016/ j.egypro.2014.11.1135.

[29] Pellegrini M, Guzzini A, Saccani e C. A preliminary assessment of the potential of low percentage green hydrogen blending in the Italian natural gas network. Energies 2020;13(21):5570. https://doi.org/10.3390/en13215570. ott.

[30] Colbertaldo P, Guandalini G, Campanari e S. Modelling the integrated power and transport energy system: the role of power-to-gas and hydrogen in long-term scenarios for Italy. Energy 2018;154:592-601. https://doi.org/10.1016/ j.energy.2018.04.089. lug.

[31] Bensmann B, Hanke-Rauschenbach R, Müller-Syring G, Henel M, Sundmacher e K. Optimal configuration and pressure levels of electrolyzer plants in context of power-togas applications. Appl Energy 2016;167:107-24. https:// doi.org/10.1016/j.apenergy.2016.01.038. apr.

[32] Pfenninger S, Staffell I. Long-term patterns of European PV output using 30 years of validated hourly reanalysis and satellite data. Energy 2016;114:1251-65. https://doi.org/ 10.1016/j.energy.2016.08.060. nov.

[33] Staffell I, Pfenninger S. Using bias-corrected reanalysis to simulate current and future wind power output. Energy 2016;114:1224-39. https://doi.org/10.1016/ j.energy.2016.08.068. nov.

[34] Adolfo D, Carcasci C. Steady-state simulation of gas distribution networks in the presence of localized hydrogen injections. In: Proceedings of 11th international conference on applied energy, Part 1, Sweden, 2019 | energy proceedings, vol. 2; 2019. p. 4.

[35] ARERA - Home page». https://www.autorita.energia.it/it/ inglese/\#.

[36] Aste N. Impact of domestic and tertiary buildings heating by natural gas in the Italian context. Energy Pol 2012;8.

[37] Alberini A, Prettico G, Shen C, Torriti e J. Hot weather and residential hourly electricity demand in Italy. Energy 2019;177:44-56. https://doi.org/10.1016/j.energy.2019.04.051. giu.

[38] Jardini JA, Tahan CMV, Gouvea MR, Ahn SU, Figueiredo e FM. Daily load profiles for residential, commercial and industrial low voltage consumers. IEEE Trans Power Deliv 2000;15(1):375-80. https://doi.org/10.1109/61.847276. gen.

[39] Federici A, Magro FD, Pozzar F, Martini e C. Analisi campionaria dei consumi elettrici e la propensione all'efficienza energetica delle PMI. 2015. https://doi.org/ 10.13140/RG.2.2.30775.83364. 
[40] Papay J. A termelestechnologiai parameterek valtozasa a gazlelepk muvelese soran. OGIL MUSZ 1968:267-73.

[41] Kohn JP. Phase equilibria and fluid properties in the chemical industry. In: Storvick TS, Sandler SI, editors. ACS symposium series 60 (1977) \$32.00. American Chemical Society; 1978. p. 537. https://doi.org/10.1002/aic.690240636. AIChE Journal, vol. 24, n. 6, pagg. 1142-1142.

[42] Ferguson JA. Gas flow in long pipelines. Chem Eng 2002:56.
[43] Poling BE, Prausnitz JM, O'Connell JP. The properties of gases and liquids. 5th ed. New York: McGraw-Hill; 2001.

[44] Schmidt M, Steinbach MC, Willert e BM. High detail stationary optimization models for gas networks. Optim Eng 2015;16(1):131-64. https://doi.org/10.1007/s11081-014-9246-x. mar.

[45] Cavana M. Biogas blending into the gas grid of a small municipality for the decarbonization of the heating sector. Biomass Bioenergy 2019:15. 